\title{
Primary Malignant Lymphoma in a Spinal Cord Presenting as an Epidural Mass with Myelopathy: A Case Report
}

\author{
Jae-Hoon Cho, Dae-Chul Cho, Joo-Kyung Sung, Kyoung-Tae Kim \\ Department of Neurosurgery, Kyungpook National University School of Medicine, Daegu, Korea
}

We report the case of a 47-year-old man who presented with progressive paraparesis and sphincter changes over 2 weeks. Magnetic resonance imaging revealed a spinal epidural mass from T9 to L2. We performed a decompressive laminectomy and mass removal. The histopathology was consistent with a small lymphocytic lymphoma. No metastatic lesion was noted in the chest and abdomen-pelvic computerized tomography (CT) and positron emission tomography computerized tomography (PET-CT) scan. The final diagnosis was primary spinal lymphoma, so we performed chemotherapy combined with radiotherapy. At one year follow-up, he had no neurological deficit and no recurrence on neurologic and radiologic exams. Primary spinal cord lymphomas should be considered in the differential diagnosis of spinal cord tumors. Early surgical management is mandatory to achieve a recovery of neurologic function, especially if the patient has a neurological deficit.

Key Words: Primary Spinal Lymphoma $\cdot$ Spinal Epidural Mass $\cdot$ Decompressive laminectomy $\cdot$ Chemo-radiotherapy

\section{INTRODUCTION}

Malignant lymphoma (ML) can involve the central nervous system either primarily or by secondary spread, which tends to occur late in the disease as part of widespread dissemination $^{8,18)}$. Lymphoma presenting as primary tumors of the spinal cord are extremely uncommon. The rate of epidural mass formation in all cases of MLs is $0.8-2.8^{6,14,19,21)}$. Owing to this rate of occurrence, a small number of research studies and case reports dominate the literature. We report a rare case of primary spinal epidural lymphoma that presented with progressive myelopathy.

\section{CASE REPORT}

A 47-year-old man presented with progressive lower extremity weakness and numbness associated with fecal and urinary incontinence over 2 weeks. The patient did not complain of fevers, night sweats, or weight loss. A physical examination

\footnotetext{
- Received: April 13, 2012 - Revised: September 4, 2012

- Accepted: September 26, 2012

Corresponding Author: Kyoung-Tae Kim, MD, PhD

Department of Neurosurgery, Kyungpook National University Hospital,

50 Samduk-2-ga, Jung-gu, Daegu 700-721, Korea

Tel: +82-53-420-5657, Fax: +82-53-423-0504

Email: nskimkt@gmail.com
}

revealed hypoesthesia on the medial aspect of both thighs with bilateral motor weakness (Gr 4-4+). Anal tone decreased and urinary incontinence was observed. The vibration senses were reduced below the T10 level. There was no abnormality of laboratory findings except the erythrocyte sedimentation rate was elevated (ESR-32 mm/h; reference value - less than $20 \mathrm{~mm} / \mathrm{h}$ ). Preoperative magnetic resonance imaging (MRI) of the thora columbar region showed an isointense spinal medulla on T1weighted images and a slightly hyperintense $\mathrm{T} 2$-weighted images of the spinal cord extending from T9-L2. The mass was of the homogeneous signal intensity and exhibited diffuse enhancement with gadolinium (Fig. 1A-E). The patient underwent a decompressive partial hemilaminectomy of T9-L2 and tumor removal. Intraoperatively, a grayish epidural mass was identified and resected to decompress the cord and cauda equina. Histopathologic analysis of the tumor specimen showed infiltration of a small lymphocytic lymphoma. Proliferation of small blastic cells, with a lymphoblastic aspect and starry sky pattern, was seen. Further immunohistochemical characterization showed that results from tumor testing were positive for $\mathrm{CD} 20, \mathrm{CD} 3$, CD5, Bcl-2, Cyclin D1 and negative for CD23, CD10 and Tcell markers (Fig. 2). We performed the further evaluations because of indicated lymphoma characteristics. The MRI of the brain and cervical spine were normal. Further work-up, including computed tomography (CT) scans of the chest and the abdomen, iliac crest bone marrow biopsy, and ultrasound of the lymph nodes showed no extramedullary lymphoma manifestation. The patient had no history of immune disorder, and 

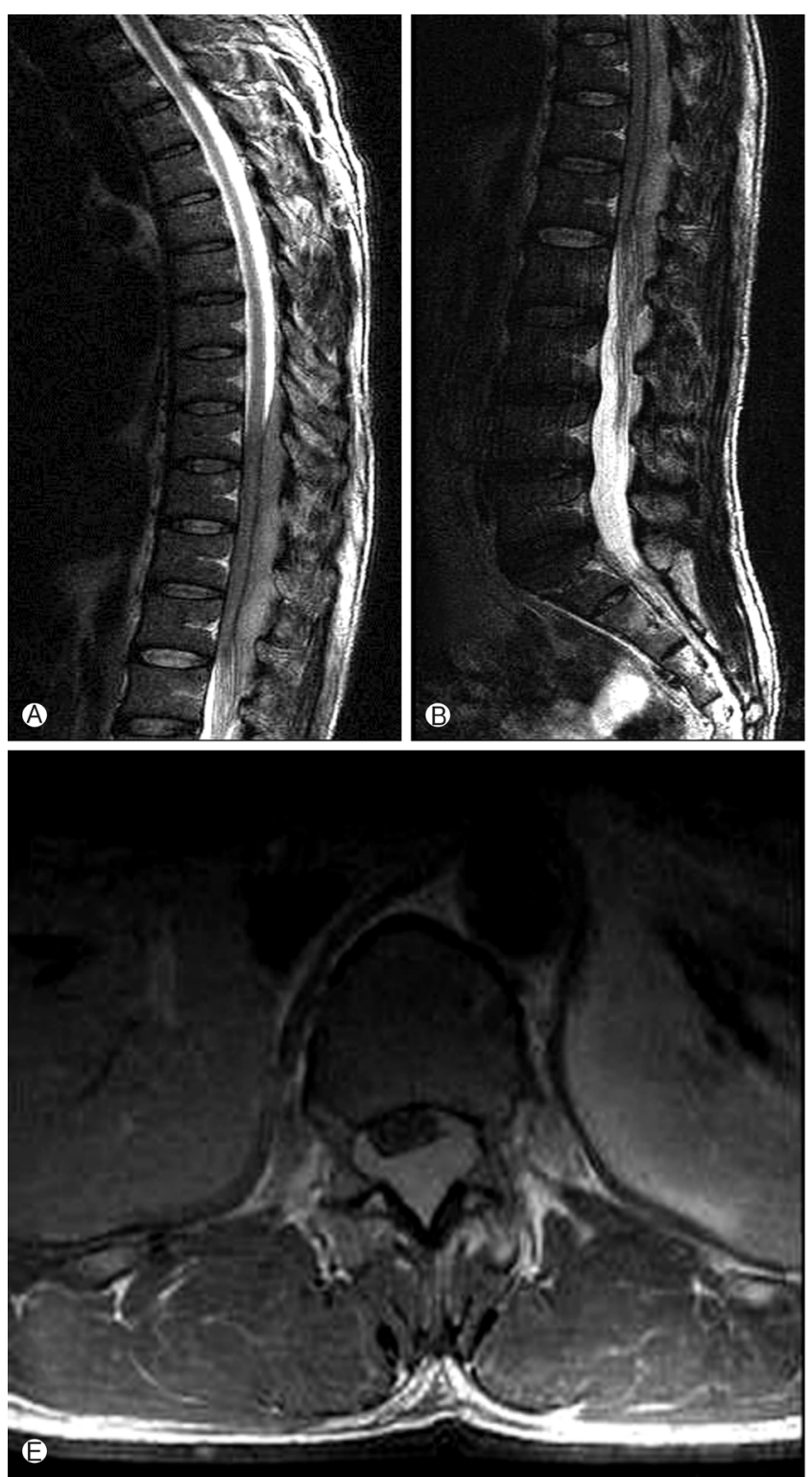

human immunodeficiency virus (HIV) testing was negative. The treatment regimen consisted of radiotherapy, $40 \mathrm{~Gy}$ administered in 20 fractions, from T9-L2, followed by combination chemotherapy with cyclophosphamide, adriamycin, oncovin, and prednisone (CHOP). A follow-up MRI one year after the surgery showed that the lesions of lymphoma had disappeared (Fig. 1F). After surgery, all preoperative symptoms completely resolved. At one year postoperatively, the patient was faring well with no evidence of local recurrence or new lesions at any other site.

\section{DISCUSSION}

Primary central nervous system lymphoma is a rare form
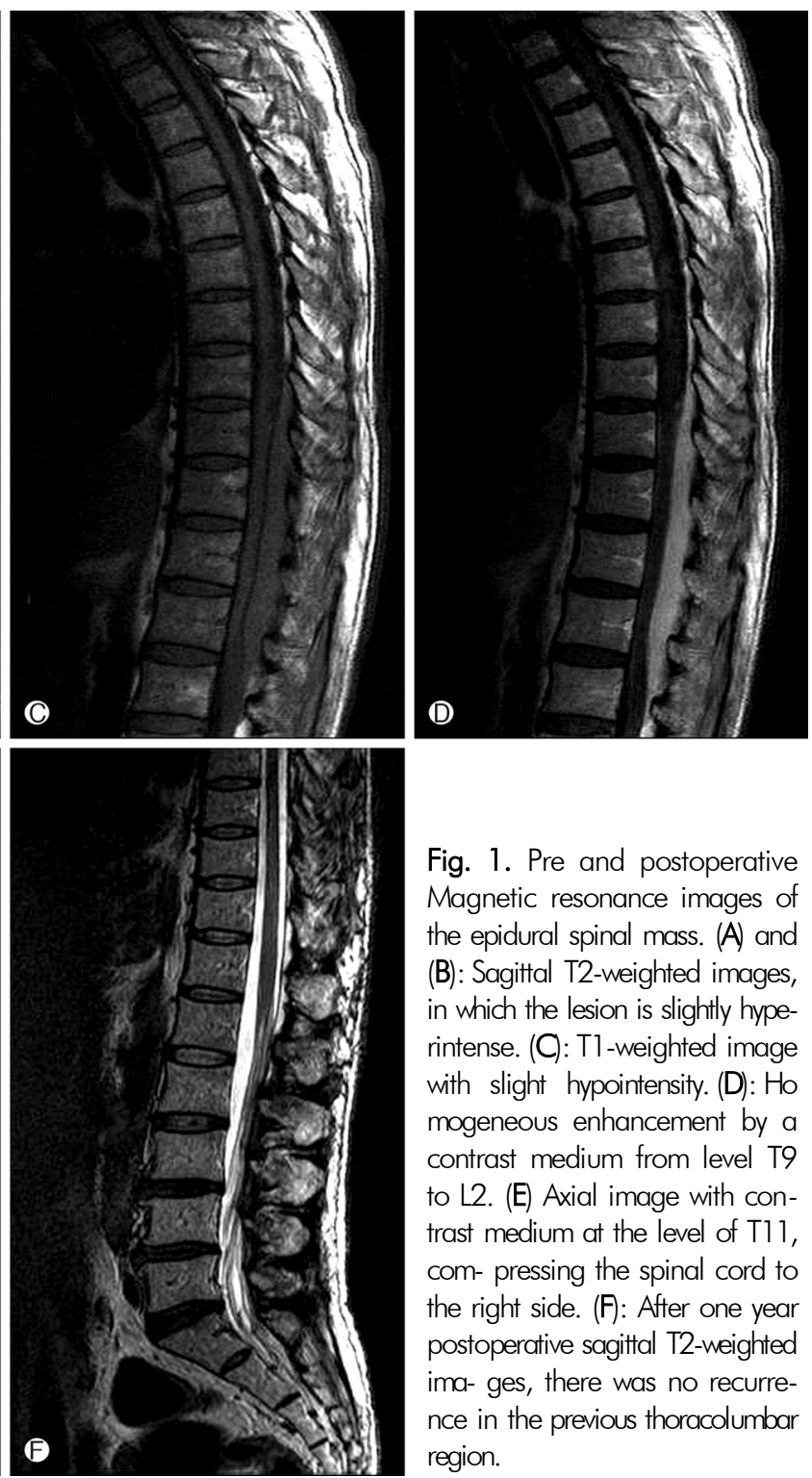

Fig. 1. Pre and postoperative Magnetic resonance images of the epidural spinal mass. (A) and (B): Sagittal T2-weighted images, in which the lesion is slightly hyperintense. (C): $\mathrm{Tl}$-weighted image with slight hypointensity. (D): Ho mogeneous enhancement by a contrast medium from level T9 to $L 2$. (E) Axial image with contrast medium at the level of $\mathrm{T} 11$, com- pressing the spinal cord to the right side. (F): After one year postoperative sagittal T2-weighted ima- ges, there was no recurrence in the previous thoracolumbar region.

of extranodal lymphoma, particularly isolated primary spinal lymphoma ${ }^{7,8)}$. Compression of the spinal cord as the first manifestation of the primary spinal lymphoma is particularly rare, with an incidence of $<5 \%{ }^{15}$. In our report, the patient initially presented with compression of the spinal cord manifesting as hypoesthesia and motor weakness of the lower extremities. Although the mechanism of epidural mass formation without systemic lymphoma has not been clarified, the origin of the mass is thought to be bone marrow ${ }^{11)}$. A small amount of lymphocytic cells can migrate to the spinal epidural space directly through the harversian canals of the vertebral bone or hematogenously via the epidural venous plexus, followed by a mass formation even before systemic lymphoma.

Findings from the CT and MRI provided only indirect diag- 
nostic evidence. On T1-weighted MRI, primary spinal lymphomas are most frequently isointense to hypointense, while T2weighted images are commonly isointense to hyperintense, with contrast enhancement ${ }^{4}$. Primary spinal lymphomas are often confused with other spinal tumors, especially metasta$\operatorname{sis}^{12)}$. In the present case the spinal lesion was originally thought to be a metastatic tumor. The MRI demonstrated an epidural mass lesion extending from T9 to $\mathrm{L} 2$, causing spinal cord compression. The lesion appeared slightly hyperintense on the T2-weighted image and exhibited continuous homogenous enhancement after gadolinium injection. The lesion was located mainly in the posterior epidural space. This is similar to the description by Negendank et al of lymphomas elsewhere in the body and $\mathrm{Li}$ et al of spinal epidural lymphomas ${ }^{12,17}$.

Most primary spinal lymphomas are comprised of diffuse large B-cell lymphomas with a minority being follicular lymphoma, precursor B-lymphoblastic lymphoma, Ki-lymphoma, diffuse lymphoblastic lymphoma, small lymphocytic lymphoma or T-cell lymphoma ${ }^{5)}$. Small lymphocytic lymphoma is composed mostly of small mature appearing lymphocytes with round nuclei and scant basophilic cytoplasm ${ }^{9)}$. In our case, immunohistochemical studies demonstrated that the tumor cells expressed the B cell markers CD19 and CD20, with coexpression of the T cell marker CD5 (Fig. 2). Expression of surface immunoglobulin $\mathrm{M}$ and immunoglobulin $\mathrm{D}$ was also noted. The tumor cells had stained positively for bcl-2 but not for CD10, CD23. Cyclin D1 staining yielded positive results for both the primary lesion and the recurrence ${ }^{1)}$. Haddad et alobserved that patients with the histopathologic subtype of mixed histiocytic and small lymphocytic lymphoma seemed to have a better survival than the other subtypes (61\% at 10 years), but a small number $(n=17)$ of patients in this group did not allow statistical confirmation of this apparent observation $^{10)}$.

In the study by Monnard et al, out of 52 patients, 48 (92\%) underwent a laminectomy, with a partial resection performed in 22 cases (42\%) and a complete resection for 7 cases (13\%). In surgery, each lesion was brownish or reddish purple and soft to firm in consistency. It does not usually adhere to the dural $\operatorname{sac}^{16,20)}$. During an operation, we performed a hemilaminectomy of T9-L2 and a partial resection of a grayish epidural mass. However, a surgical resection does not have the survival benefits and the reported median overall survival durations with surgery alone are similar to those of untreated patients ${ }^{2,3)}$. Some reports suggest that well-established treatments, such as radiotherapy and chemotherapy or a combination of both should remain as the mainstays and that surgery should be used in combination with radiotherapy and/or chemotherapy ${ }^{13)}$.

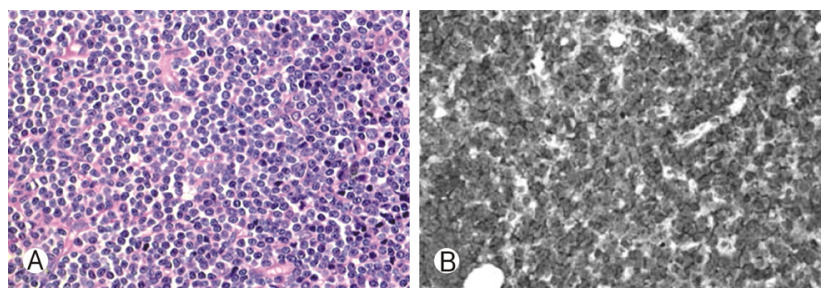

Fig. 2. (A) Photomicrograph of a monomorphous specimen and comprised of small lymphocytes with a blend cytological appearance. A heterogenous mixture of cells including prolymphocytes, lymphoplasmacytoid cells or features of disease progression with an increased number of larger cells (paraimmunoblasts) and mitoses or an aberrant phenotype should be considered in the small lymphocytic lymphoma (Haematoxylin and eosin, $\times 400$.) (B) Immunohistoche- mical staining showing tumor cells positive for CD20 (EnVision, original magnification $\times 200$ ).

Therefore, concurrent chemoradiotherapy treatment was provide (CHOP; cyclophosphamide, adriamycin, oncovin, and prednisone+radiotherapy; $40 \mathrm{~Gy}$ administered in 20 fractions) after pathologic confirmation. Monnard et al observed local control of $88 \%$ and a 5 -year overall survival of $69 \%$ with combined modality treatment. In a multivariate analysis, they found that the combined modality treatment was statistically superior to RT alone ${ }^{16}$. Also, they reported a post-treatment complete neurologic response in $25 \%$ of patients who had initial motor deficits. The remaining $75 \%$ had partial recovery. In their multivariate analysis, they have found that complete neurologic response was the most significant favorable prognostic finding with regard to overall survival $(\mathrm{p}=$ $0.001)^{16)}$. In the present case, because of the manifestations of compression, timely surgical decompression was required to allow for a recovery of nerve function even before the diagnosis had been made. After surgery, the neurologic function in our patient was completely recovered. Therefore, every effort should be made to recognize and treat this disease appropriately.

\section{CONCLUSION}

Primary small lymphocytic lymphomas are rare, especially with an initial presentation of paraparesis. A primary small lymphocytic lymphoma of the spine should be considered in the differential diagnosis of primary spine tumors. Surgical decompression and tumor removal are mandatory to recover neurologic function, if the patient had a neurologic deficit. The combined modality treatment after surgical decompression resulted in improvement of the neurologic symptoms and remission of the lymphoma. 


\section{REFERENCES}

1. Barnard M, Perez-Ordonez B, Rowed DW, Ang LC: Primary spinal epidural mantle cell lymphoma: case report. Neurosurgery 47:1239-1241, 2000

2. Batchelor T, Loeffler JS: Primary CNS lymphoma. J Clin Oncol 24:1281-1288, 2006

3. Bellinzona M, Roser F, Ostertag H, Gaab RM, Saini M: Surgical removal of primary central nervous system lymphomas (PCNSL) presenting as space occupying lesions: a series of 33 cases. Ejso 31:100-105, 2005

4. Boukobza M, Mazel C, Touboul E: Primary vertebral and spinal epidural non-hodgkin's lymphoma with spinal cord compression. Neuroradiology 38:333-337, 1996

5. Chiodo A: Spinal cord injury caused by epidural B-cell lymphoma: report of two cases. J Spinal Cord Med 30:70-72, 2007

6. Lee DY, Chung CK, Jahng TA, Kim HJ: Treatment of thoracolumbar junction metastasis: does posterior approach suffice? Korean J Spine 1:207-211, 2004

7. Ebus SC, Bernsen HJ, Norel Van GJ, Donk R: Primary nonhodgkin's lymphoma in multiple vertebrae presenting as a lumbar radicular syndrome: a case report. Spine (Phila Pa 1976) 27:E271-273, 2002

8. Friedman M, Kim TH, Panahon AM: Spinal cord compression in malignant lymphoma. Treatment and results. Cancer 37:14851491, 1976

9. Gupta D, Lim MS, Medeiros LJ, Elenitoba-Johnson KS: Small lymphocytic lymphoma with perifollicular, marginal zone, or interfollicular distribution. Mod Pathol 13:1161-1166, 2000

10. Haddad P, Thaell JF, Kiely JM, Harrison EG, Miller RH: Lymphoma of the spinal extradural space. Cancer 38:1862-1866, 1976

11. Higashida T, Kawasaki T, Sakata K, Tanabe Y, Kanno H, Yama- moto I: Acute lymphocytic leukemia recurring in the spinal epidural space. Neurol Med Chir (Tokyo) 47:375-378, 2007

12. Li MH, Holtas S, Larsson EM: MR imaging of spinal lymphoma. Acta Radiol 33:338-342, 1992

13. Liu BL, Cheng JX, Zhang X, Zhang W, Cheng H: Limited role of surgery in the management of primary central nervous system lymphoma (Review). Oncol Rep 22:439-449, 2009

14. Mak KS, Lee LK, Mak RH, Wang S, Pile-Spellman J, Abrahm $\mathrm{JL}$, et al: Incidence and treatment patterns in hospitalizations for malignant spinal cord compression in the United States, 1998-2006. Int J Radiat Oncol Biol Phys 80:824-831, 2011

15. McDonald AC, Nicoll JA, Rampling RP: Non-hodgkin's lymphoma presenting with spinal cord compression; a clinicopathological review of 25 cases. Eur J Cancer 36:207-213, 2000

16. Monnard V, Sun A, Epelbaum R, Poortmans P, Miller RC, Verschueren T, et al: Primary spinal epidural lymphoma: patients' profile, outcome, and prognostic factors: a multicenter Rare Cancer Network study. Int J Radiat Oncol Biol Phys 65:817823, 2006

17. Negendank WG, al-Katib AM, Karanes C, Smith MR: Lymphomas: MR imaging contrast characteristics with clinical-pathologic correlations. Radiology 177:209-216, 1990

18. Pels H, Vogt I, Klockgether T, Schlegel U: Primary non-hodgkin's lymphoma of the spinal cord. Spine (Phila Pa 1976) 25: 2262-2264, 2000

19. Perry JR, Deodhare SS, Bilbao JM, Murray D, Muller P: The significance of spinal cord compression as the initial manifestation of lymphoma. Neurosurgery 32:157-162, 1993

20. Samadian M, Vahidi S, Khormaee F, Ashraf H: Isolated, primary spinal epidural hodgkin's disease in a child. Pediatr Neurol 40:480-482, 2009

21. Zheng JS, Wang M, Wan S, Zhou YQ, Yan M, Chen QF, et al: Isolated primary non-hodgkin's lymphoma of the thoracic spine: a case report with a review of the literature. J Int Med Res 38:1553-1560, 2010 